\title{
Mimic Mini-laparoscopic Surgery is a Simple and Secure Approach Using Direct Placement of 3-mm Trocars without Noticeable Scars
}

\author{
Masao Ichikawa, Shigeo Akira, Eri Hamano, \\ Shuichi Ono, Katsuya Mine and Toshiyuki Takeshita \\ Department of Obstetrics and Gynecology, Nippon Medical School
}

\begin{abstract}
Minimally invasive surgery is widely used in gynecology. Women who seek a cosmetic advantage (i.e., concealed scars) choose minimally invasive surgery. Although laparoendoscopic single-site surgery could be an ideal solution, some of our patients have had cosmetic problems, such as pigmentation and cicatrix of the umbilicus. In addition, umbilical eversion and umbilical herniation occasionally develop. Therefore, mini-laparoscopic surgery using 3-mm trocars can be recommended for patients who do not want the natural appearance of the navel to be altered. We have developed an approach to achieve a superior cosmetic outcome by direct placement of 3-mm trocars in the lateral wall of the abdomen and at the lower border of the pubic hair. We refer to this method as mimic mini-laparoscopic surgery and report cases in which this procedure was used.
\end{abstract}

(J Nippon Med Sch 2013; 80: 78-82)

Key words: mini-laparoscopic surgery, minimally invasive surgery, ovarian cystectomy, laparoendoscopic single-site surgery

\section{Introduction}

There are 2 main methods to achieve good cosmetic outcomes with laparoscopic surgery for gynecologic diseases: laparoendoscopic single-site surgery (LESS), in which multiple trocars ${ }^{1,2}$ or a multichannel platform ${ }^{3.4}$ through the umbilicus is used, and mini-laparoscopic surgery ${ }^{5,6}$ (also known as micro-laparoscopic surgery ${ }^{7}$ ), in which smaller ports to accommodate a $3-\mathrm{mm}$ forceps or a $2-\mathrm{mm}$ needle forceps are used.

Of these 2 methods, LESS appears to be the more promising for achieving good cosmetic outcomes for gynecologic surgery, the indications for which have expanded from benign ovarian cysts to gynecologic malignancies ${ }^{8}$, based on the technical skills of the surgeon. However, we have recently treated several patients who had cosmetic problems, such as pigmentation and cicatrix of the umbilicus, after LESS (Fig. la and b). These problems are presumably caused by compression by trocars inserted into the umbilicus. We have found few published reports of such problems, but our experiences suggest that their incidence might be higher than commonly believed. In addition, other complications, such as umbilical eversion and umbilical herniation ${ }^{9}$, can also occur. These

Correspondence to Masao Ichikawa, MD, PhD, Department of Obstetrics and Gynecology, Nippon Medical School, 11-5 Sendagi, Bunkyo-ku, Tokyo 113-8603, Japan

E-mail: masai@nms.ac.jp

Journal Website (http://www.nms.ac.jp/jnms/) 

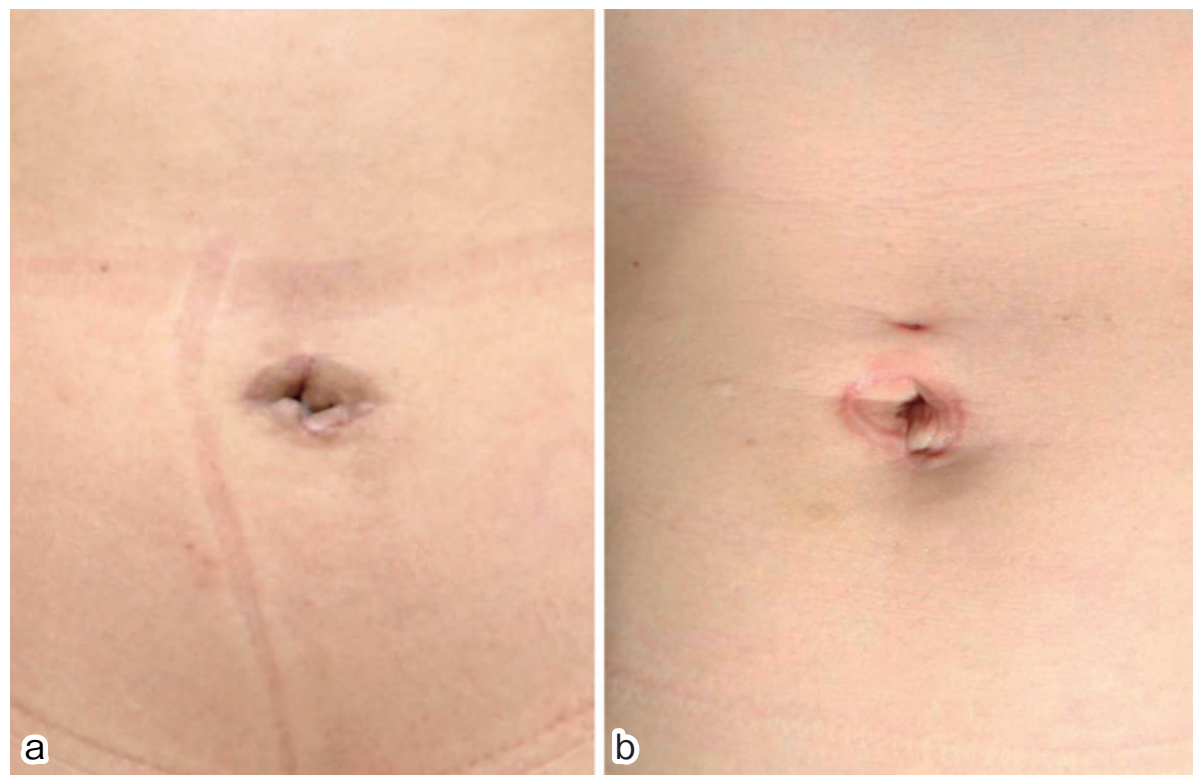

Fig. 1 An umbilicus with cosmetic problems after LESS.

a) Pigmentation of the umbilicus after LESS. b) A round cicatrix of the umbilicus after LESS. This patient has a pinhole above the umbilicus for a navel ring. The incision of the umbilicus for LESS was made in a way to preserve the pinhole.

complications are not trivial, because they can cause irreversible changes to the appearance of the umbilicus. Therefore, we must exercise care when performing LESS for patients who do not want the appearance of the navel to be altered. For such patients, we should choose another surgical approach that has a lower risk of the aforementioned complications. Indeed, minilaparoscopic surgery might be a good choice for patients who want to preserve the natural appearance of the umbilicus. Because the trocars for mini-laparoscopic surgery leave noticeable scars in the abdominal wall, we have developed a modification of this surgery that achieves better cosmetic outcomes. When 3-mm trocars are placed in the lateral wall of the abdomen and at the lower border of the pubic hair, all trocar scars can be hidden or resemble a lentigo and eventually become unnoticeable. Thus, we refer to this method as mimic mini-laparoscopic surgery.

\section{Case Reports}

\section{Case 1}

The patient was 34-year-old nullipara. She had an 8-year history of endometriosis and had taken low- dose oral contraceptives for 7 years. She sought evaluation for dysmenorrhea and was found to have a 4.5 -cm right ovarian cyst.

The patient was placed in the Trendelenburg position with a 20- to 30-degree tilt and inclined to the right to displace the bowel toward the right side to avoid bowel damage during the insertion of the trocars. The trocar positions are shown in Figure 2a and $\mathbf{b}$. One 3-mm trocar was placed in the left lateral abdominal wall, and two 3-mm trocars were placed within the pubic hair. For the laparoscope a 5 -mm trocar was placed in the umbilicus, and a $12-\mathrm{mm}$ trocar was inserted into the vagina with a uterine manipulator to introduce the retrieval bag for the resected tissues, as is done during natural orifice transluminal endoscopy ${ }^{10}$. The operator stood on the left side of the body and used 2 working channels (Fig. 2a).

Because the right uterosacral ligament and right ovary had mild adhesions (Fig. 2c), adhesiolysis and right ovarian cystectomy were performed. The resected tissue was removed in a $10-\mathrm{cm}$ retrieval bag through the 12-mm vaginal trocar. At the end of the procedure, laparoscopic uterosacral nerve ablation was performed. The total operative time was 172 minutes, and total blood loss was $20 \mathrm{~mL}$. No 


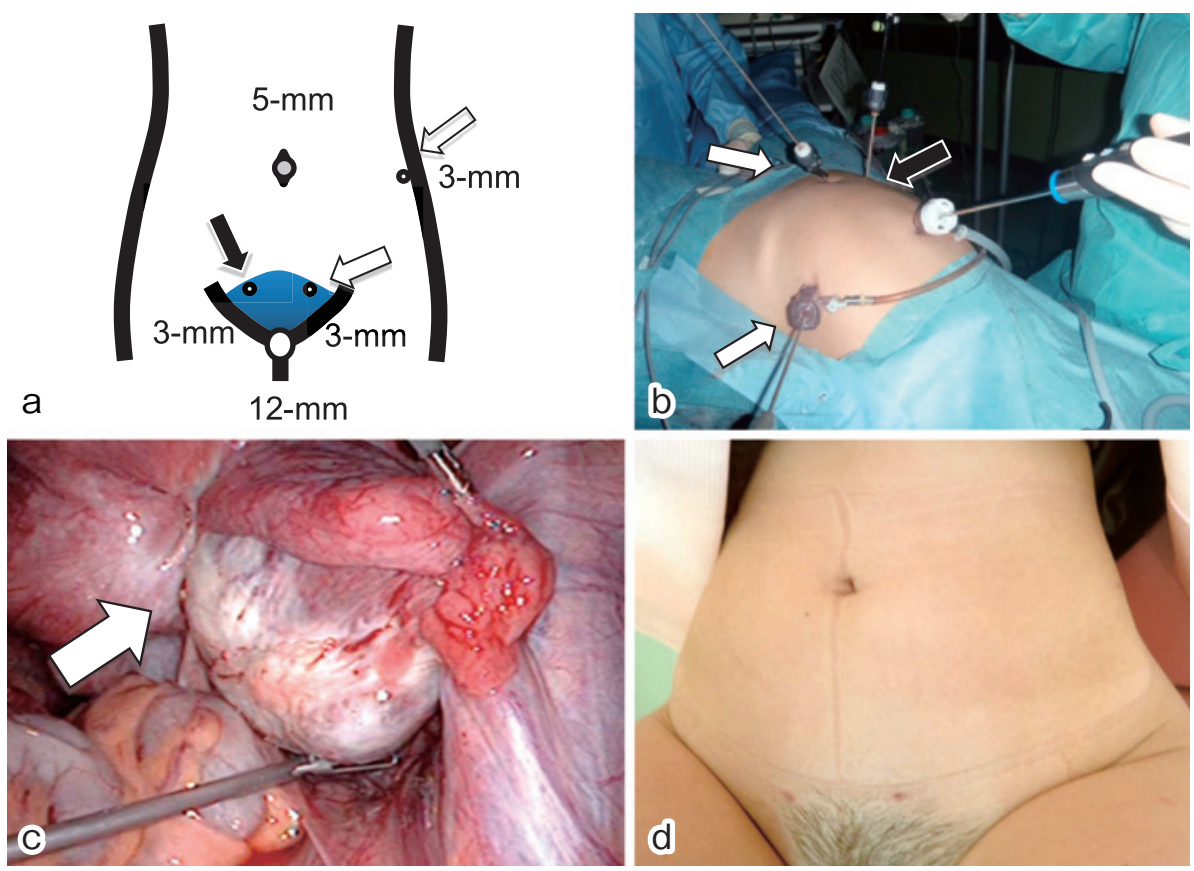

Fig. 2 Mimic mini-laparoscopic surgery with three 3-mm trocars. a) and b) The diagram indicates the positioning of the three 3-mm trocars and the $12-\mathrm{mm}$ trocar. The operator stands on the left side of the patient and uses 2 working channels (white arrows), and the assistant stands on the right side and controls the laparoscope and third forceps (black arrow). c) The enlarged right ovary with adhesions to the posterior of the uterus. d) The trocar scars 1 month later.

perioperative complications occurred. The patient's scar are shown in Figure 2d $(1$ month postoperatively).

\section{Case 2}

The patient was a 23-year-old nullipara. She sought evaluation for an asymptomatic 6-cm right ovarian dermoid.

The specific details of the operation were identical to that of case 1 except for the use of a 2-mm loop retractor (Mini Loop Retractor II; Covidien, Mansfield, MA, USA), which was placed in the right lower quadrant of the abdominal wall (Fig. 3a, b and c).

The right ovarian cyst was suspended with a 2mm loop retractor (Fig. 3c) and was then resected. The resected tissue was removed in the same fashion as described for case 1 . The total operative time was 114 minutes, and the total blood loss was 0 mL. No perioperative complications occurred. The trocar scars were not prominent, even immediately after surgery (Fig. 3d).

\section{Case 3}

The patient was a 35-year-old nullipara at 6 weeks' gestation following assisted reproduction (freeze-thaw blastocyst transfer). An ectopic pregnancy in the right fallopian tube was diagnosed.

The specific details of the operation were identical to that of case 1, with the exception that two 3-mm trocars were used (Fig. 4a). The swollen right fallopian tube was resected and retrieved in the same fashion as described for case 1. The total operative time was 70 minutes, and total blood loss was $0 \mathrm{~mL}$. No perioperative complications occurred. The trocar scars were not prominent (Fig. $4 \mathbf{b}$ ).

\section{Discussion}

There are 3 ways to make trocar scars less noticeable: centralization of the trocars into the umbilicus (as in LESS); reduction of the size of the trocar (as in mini-laparoscopic surgery); and use of the vaginal route for trocar placement (as in natural orifice transluminal endoscopy) ${ }^{10}$. In the present report, we have introduced a fourth approach (mimic 

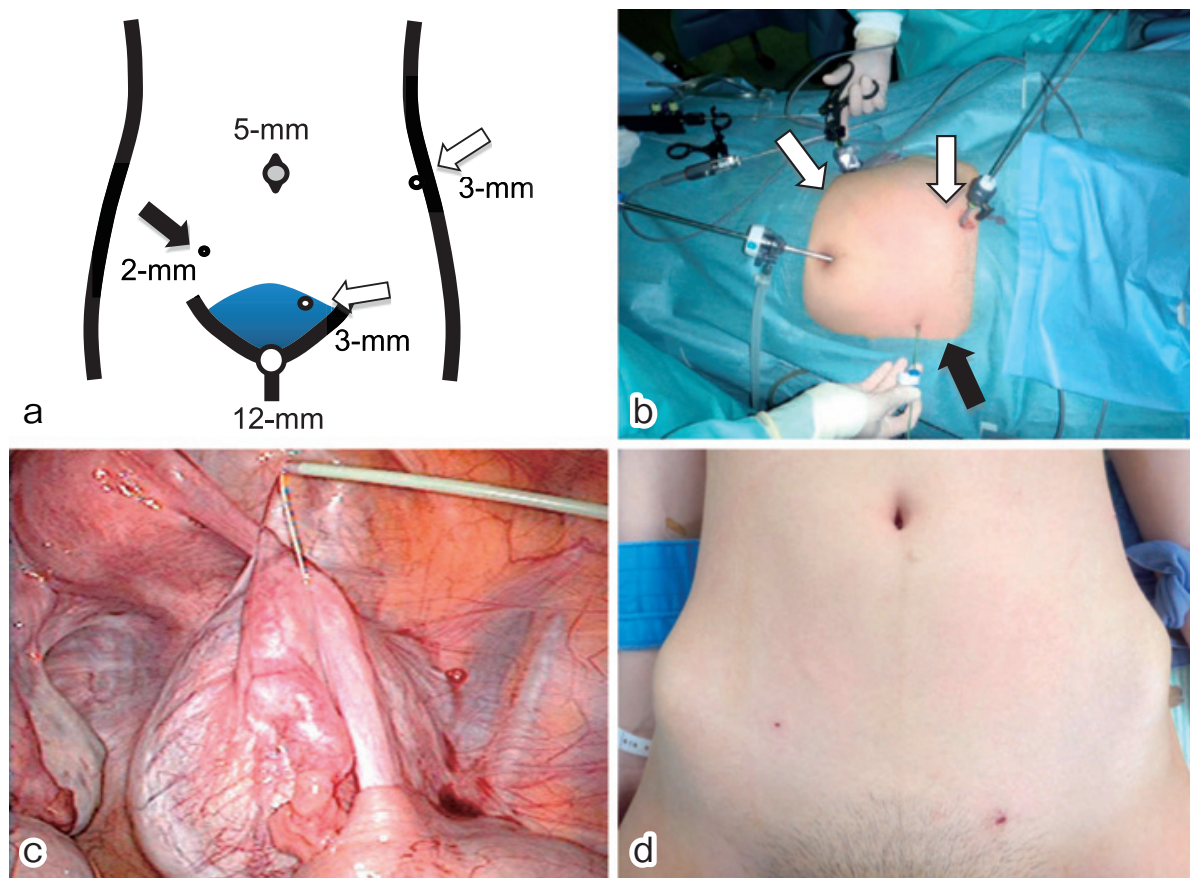

Fig. 3 Mimic mini-laparoscopic surgery with two 3-mm trocars and a 2-mm endo surge retractor. a-c) The diagram indicates the positioning of the two 3-mm trocars, the 2-mm loop retractor, and the $12-\mathrm{mm}$ trocar. The operator stands on the left side of the patient and uses 2 working channels (white arrows), and the assistant stands on the right side and controls the laparoscope and the 2-mm loop retractor (black arrow). c) An enlarged right ovary suspended with a 2-mm loop retractor. d) The patient's scar immediately after surgery.

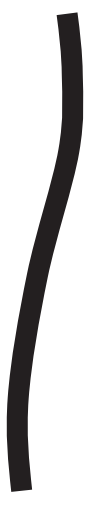

a
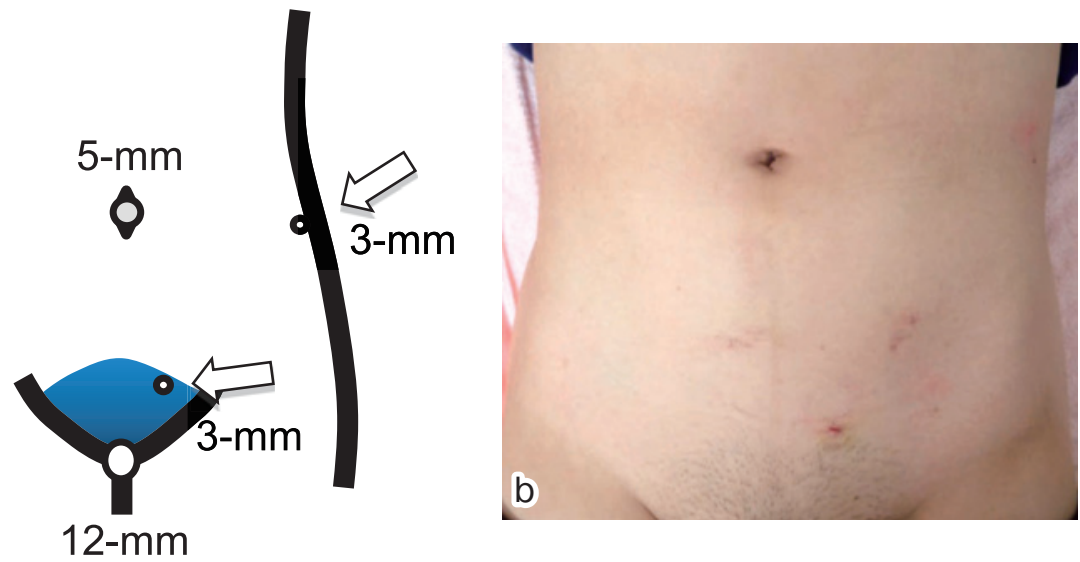

Fig. 4 Mimic mini-laparoscopic surgery with two 3-mm trocars. The diagram indicates the positioning of the two 3-mm trocars and the 12-mm trocar. The operator stands on the left side of the patient and uses 2 working channels (white arrows). b) The scars immediately after surgery.

trocar position).

To date, 2 conventional trocar positions have been available in Japan: the diamond trocar position (Fig. 5a) and the ipsilateral trocar position (Fig. 5b). Both positions can be used for mini-laparoscopic surgery or conventional 5-mm laparoscopic surgery. However, from a cosmetic point of view, the linearly arrayed trocar scars remain strikingly visible, like the asterism Orion's belt, which comprises 3 secondmagnitude stars (Fig. 5a). In the mimic trocar position, 3-mm trocars are placed on the lateral abdominal wall and within the pubic hair of the lower abdomen, so those scars are less prominent and unnoticeable (Fig. 5c and d). Thus, we 


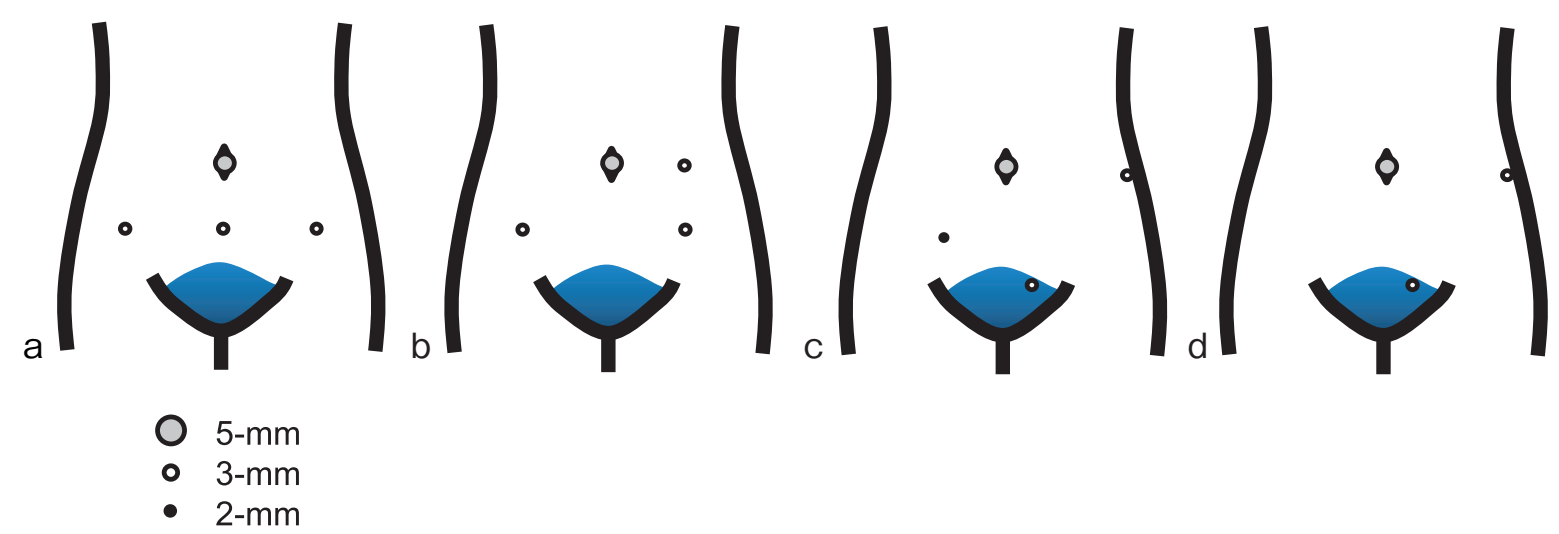

Fig. 5 Comparison of the various trocar positions. a) Diamond trocar position. b) Ipsilateral trocar position. c) Mimic trocar position with two 3-mm trocars and a 2-mm loop retractor. d) Mimic trocar position with two 3-mm trocars.

developed a better cosmetic surgery, i.e., the mimic mini-method.

\section{References}

We have presented 3 types of mimic minilaparoscopic operations: the mimic mini-method with three 3-mm trocars; the mimic mini-method with two 3-mm trocars and a 2-mm needle device; and the mimic mini-method with two 3-mm trocars only. The first and second methods are suitable for unilateral ovarian cystectomy, such as for endometrial or dermoid cysts, for which a third forceps is needed for fine dissection, and strong traction is needed to preserve ovarian function as much as possible. The third method is for simpler operations, such as tubectomy for treating ectopic pregnancy or unilateral oophorectomy for treating small ovarian cysts. If the patient has bilateral lesions or adhesions, conventional trocar positions are a better choice because approaching both lesions and increasing the size of a trocar from $3 \mathrm{~mm}$ to a 5 or $12 \mathrm{~mm}$ are easier.

Another advantage of mimic mini-laparoscopic surgery is that it is easy to perform, because the special instruments used in LESS are not needed and because the triangulation for manipulating instruments is sufficient. With these benefits, surgery is safe and secure, and good cosmetic outcomes are achieved. This surgery also does not require a long learning curve.

Mimic mini-laparoscopic surgery has cosmetic advantages for patients who do not want alterations to the navel and is an alternative approach to LESS.

1. Sinha R, Sundaram M, Mahajan C, et al.: Singleincision total laparoscopic hysterectomy. J Minim Access Surg 2011; 7: 78-82.

2. Ichikawa M, Akira S, Mine K, et al.: Evaluation of laparoendoscopic single-site gynecologic surgery with a multitrocar access system. J Nihon Med Sch 78: $235-240$.

3. Uppal S, Frumovitz M, Escobar P, Ramirez PT: Laparoendoscopic single-site surgery in gynecology: review of literature and available technology. J Minim Invasive Gynecol 2011; 18: 12-23.

4. Fader AN, Levinson KL, Gunderson CC, Winder AD, Escobar PF: Laparoendoscopic single-site surgery in gynaecology: A new frontier in minimally invasive surgery. J Minim Access Surg 2011; 7: 71-77.

5. Novitsky YW, Kercher KW, Czerniach DR, et al.: Advantages of mini-laparoscopic vs conventional laparoscopic cholecystectomy: results of a prospective randomized trial. Arch Surg 2005; 140: $1178-1183$

6. Mostafa G, Matthews BD, Sing RF, Kercher KW, Heniford BT: Mini-laparoscopic versus laparoscopic approach to appendectomy. BMC Surg 2001; 1: 4.

7. Siegle JC, Bishop LJ, Rayburn WF: Randomized comparison between two microlaparoscopic techniques for partial salpingectomy. JSLS 2005; 9: 30-34.

8. Fader AN, Escobar PF: Laparoendoscopic single-site surgery (LESS) in gynecologic oncology: technique and initial report. Gynecol Oncol 2009; 114: 157-161.

9. Gunderson CC, Knight J, Ybanez-Morano J, et al:: The risk of umbilical hernia and other complications with Laparoendoscopic Single-Site Surgery. J Minim Invasive Gynecol 2012; 19: 40-45.

10. Tsin DA, Colombero LT, Lambeck J, Manolas P: Minilaparoscopy-assisted natural orifice surgery. JSLS 2007; 11: 24-29.

(Received, February 7, 2012)

(Accepted, $\quad$ May 10, 2012) 